D.T. Wickramasinghe, L. Ferrario, and G.V. Bicknell, eds.

\title{
Order and Chaos in Accretion Disks of Active Galactic Nuclei
}

\author{
Abraham C.-L. Chian \\ National Institute for Space Research - INPE, P.O. Box 515, 12201-970 \\ São José dos Campos - SP, Brazil
}

\begin{abstract}
Langmuir turbulence plays an important role in electron heating and generation of plasma emission in accretion disks of active galactic nuclei. The nonlinear dynamical behavior of Langmuir turbulence and its relevance in the interpretation of AGN variability is discussed. In particular, we study nonlinear saturation of the Langmuir stimulated modulational instability, for which the low-frequency mode is a resonant ion-acoustic wave. The nonlinear system of coupled wave equations is shown to undergo transition from order to chaos via the route of quasiperiodicity. The periodic, quasiperiodic and chaotic variabilities in AGN emissions may be the electromagnetic signatures of the ordered and chaotic states of Langmuir turbulence in accretion disks or jets of AGN.
\end{abstract}

The study of AGN variability can provide major clues to plasma processes such as Langmuir turbulence operating in AGN source region. Langmuir turbulence can cause electron heating and nonthermal plasma emissions (from radio to $\gamma$-ray) in accretion disks and jets of AGN (see e.g., Baker et al. 1988, Lesch 1991, Chian 1996). For example, electron heating in accretion disks of AGN can be produced by Langmuir turbulence excited by the energetic electron beams emerging from magnetic reconnection regions (Lesch 1991); collective emissions of electromagnetic waves can occur when relativistic electrons pass through a Langmuir turbulence wave field in astrophysical jets (Baker et al. 1988).

In this paper, we investigate the nonlinear dynamical behavior of Langmuir turbulence in accretion disks and jets of AGN paying special attention to the Langmuir stimulated modulational instability, in the absence of driving and dissipation. We show that such a Hamiltonian system exhibits transition from order to chaos via the route of quasiperiodicity.

The electrostatic Langmuir parametric instability contains basically three distinct regimes (Chian 1996): PDI (parametric decay instability), OTSI (oscillating two-stream instability), and SMI (stimulated modulational instability). In the PDI, a Langmuir pump wave decays into a Stokes Langmuir wave and an ion-acoustic daughter wave. In the OTSI, a Langmuir pump wave interacts with purely growing density perturbations to excite a pair of Stokes and anti-Stokes Langmuir waves. The SMI can be regarded as a four-wave decay instability involving the pump, Stokes, and anti-Stokes Langmuir waves, along with an ionacoustic daughter wave whose real frequency is on the order of the ion-acoustic frequency (i.e., a normal plasma mode). A three-dimensional linear stability analysis of Langmuir parametric instabilities shows that the maximum growth 
rate of the SMI is comparable to the PDI and OTSI, albeit the wavenumber for which the maximum growth occurs is determined by a four-wave interaction wherein the anti-Stokes mode is resonant (Bardwell \& Goldman 1976). The OTSI is very sensitive to the magnetic field; in contrast, the SMI and PDI are not much affected by the presence of a weak magnetic field (Weatherall et al. 1981). Observational evidence of nonlinear decay and modulational processes associated with Langmuir turbulence has been obtained by in situ measurement of spacecrafts in solar wind and planetary bow shocks (Chian \& Alves 1988, Chian \& Abalde 1995).

The one-dimensional nonlinear coupling of Langmuir and ion-acoustic waves is governed by the electrostatic Zakharov equations (Zakharov 1972; Chian \& Rizzato 1994)

$$
\begin{gathered}
\left(\partial_{t}^{2}-3 v_{t h}^{2} \partial_{x}^{2}+\omega_{p}^{2}\right) E=-\frac{\omega_{p}^{2}}{n_{0}} n E, \\
\left(\partial_{t}^{2}-v_{S}^{2} \partial_{x}^{2}\right) n=\frac{\varepsilon_{0}}{2 m_{i}} \partial_{x}^{2}\left\langle E^{2}\right\rangle,
\end{gathered}
$$

where $E$ is the Langmuir electric field, $n$ is the ion density fluctuation, $\omega_{p}=$ $\left(n_{0} e^{2} / m_{e} \varepsilon_{0}\right)^{1 / 2}$ is the electron plasma frequency, $v_{t h}=\left(K T_{e} / m_{e}\right)^{1 / 2}$ is the electron thermal velocity, $v_{S}=\left[K\left(T_{e}+T_{i}\right) / m_{i}\right]^{1 / 2}$ is the ion-acoustic velocity and the angular brackets denote averaging over the fast timescale. To study the fourwave process $L_{0} \rightleftharpoons L_{-}+L_{+}+S$, we write the total Langmuir electric field as $E=E_{0}+E_{-}+E_{+}$, with the subscript $\alpha=(0,-,+)$ referring to the pump, Stokes and anti-Stokes Langmuir waves, respectively. We adopt the modulational representation $E_{\alpha}(x, t)=(1 / 2) \mathcal{E}_{\alpha}(x, t) \exp i\left(k_{\alpha} x-\omega_{\alpha} t\right)+c . c$. , where $\mathcal{E}_{\alpha}(x, t)$ is a slowly varying complex envelope such that $\left|\partial_{x}^{2} \mathcal{E}_{\alpha}\right| \ll\left|k_{\alpha} \partial_{x} \mathcal{E}_{\alpha}\right|$ and $\left|\partial_{t}^{2} \mathcal{E}_{\alpha}\right| \ll\left|\omega_{\alpha} \partial_{t} \mathcal{E}_{\alpha}\right|$; and $\omega_{\alpha}^{2}=\omega_{p}^{2}+3 v_{t h}^{2} k_{\alpha}^{2}$. Likewise, we write $n(x, t)=(1 / 2) \mathrm{n} \exp i\left(k_{S} x-\omega_{S} t\right)+$ c.c., with $\omega_{S}^{2}=v_{S}^{2} k^{2}$. The four-wave process under study involves the coupling of two wave triplets which satisfy the following phase-matching conditions

$$
\omega_{\mp} \approx \omega_{0} \mp \omega_{S}, \quad k_{\mp}=k_{0} \mp k_{S} .
$$

We shall assume perfect wave-vector matching, but imperfect frequency matching.

An introduction of the modulational representation into Eqs. (1) -(2) gives the following complex dynamical system

$$
\begin{gathered}
\partial_{t} \mathcal{E}_{0}=c_{-} \omega_{1}\left(\mathcal{E}_{S} \mathcal{E}_{-} \exp i \delta_{-} t-r \mathcal{E}_{S}^{*} \mathcal{E}_{+} \exp i \delta_{+} t\right), \\
\partial_{t} \mathcal{E}_{S}=-c_{-}\left(m_{e} \omega_{p}^{2} / 2 m_{i} \omega_{S}\right)\left(\mathcal{E}_{0} \mathcal{E}_{-}^{*} \exp -i \delta_{-} t+r \mathcal{E}_{0}^{*} \mathcal{E}_{+} \exp i \delta_{+} t\right), \\
\partial_{t} \mathcal{E}_{-}=-c_{-} \omega_{-} \mathcal{E}_{0} \mathcal{E}_{S}^{*} \exp -i \delta_{-} t, \\
\partial_{t} \mathcal{E}_{+}=c_{+} \omega_{+} \mathcal{E}_{0} \mathcal{E}_{S} \exp -i \delta_{+} t,
\end{gathered}
$$

where $\mathcal{E}_{S}$ is the ion-acoustic electric field; the coupling coefficients are given by $c_{-}=\left(e k_{S}\right) /\left(4 m_{e} \omega_{0} \omega_{-}\right)$and $c_{+}=\left(e k_{S}\right) /\left(4 m_{e} \omega_{0} \omega_{+}\right) ; \delta_{-}=\omega_{0}-\omega_{S}-\omega_{-}$and $\delta_{+}=\omega_{0}+\omega_{S}-\omega_{+}$are the parameters of linear frequency mismatch for each of two wave triplets. The ratio $r$, which measures the relative coupling strength of anti-Stokes and Stokes modes, is defined as $r=c_{+} / c_{-}$. 
The linear stability analysis of the Langmuir stimulated modulational instability can be performed by assuming $\partial_{t} \mathcal{E}_{0}=0$ and $\left|\mathcal{E}_{0}\right| \gg\left|\mathcal{E}_{ \pm}\right|,\left|\mathcal{E}_{S}\right|$. A Fourier analysis of Eqs. (5)-(7) then yields a nonlinear dispersion relation whose solution gives a growth rate $\Gamma=(\sqrt{3} / 2)\left[\left(c_{S} c_{0} \omega_{0}\left|\mathcal{E}_{0}\right|^{2}\right) /\left(2 \omega_{+} \omega_{-}\right)\right]^{1 / 3}$.

The analysis of nonlinear solutions of Eqs. (4)-(7) is simplified by defining $\mathcal{E}_{\alpha}=\beta_{\alpha} F_{\alpha}^{1 / 2} \exp i \phi_{\alpha}$, where the amplitude $F_{\alpha}$ and phase $\phi_{\alpha}$ are real variables, $\beta_{\alpha}$ are complex normalization constants. Substituting this polar representation into Eqs. (4)-(7) leads to the following six degree-of-freedom system

$$
\begin{gathered}
\dot{F}_{1}=2\left(F_{1} F_{2} F_{3}\right)^{1 / 2} \cos \phi_{-}-2 r\left(F_{1} F_{2} F_{4}\right)^{1 / 2} \cos \phi_{+}, \\
\dot{F}_{2}=-2\left(F_{1} F_{2} F_{3}\right)^{1 / 2} \cos \phi_{-}-2 r\left(F_{1} F_{2} F_{4}\right)^{1 / 2} \cos \phi_{+}, \\
\dot{F}_{3}=-2\left(F_{1} F_{2} F_{3}\right)^{1 / 2} \cos \phi_{-}, \\
\dot{F}_{4}=2 r\left(F_{1} F_{2} F_{4}\right)^{1 / 2} \cos \phi_{+}, \\
\dot{\phi}_{-}=(1 / 2)\left(H+\delta_{-}^{\prime} F_{3}+\delta_{+}^{\prime} F_{4}\right)\left(1 / F_{2}-1 / F_{1}\right)+\left(F_{1} F_{2} / F_{3}\right)^{1 / 2} \sin \phi_{-}-\delta_{-}^{\prime}, \\
\dot{\phi}_{+}=(1 / 2)\left(H+\delta_{-}^{\prime} F_{3}+\delta_{+}^{\prime} F_{4}\right)\left(-1 / F_{2}-1 / F_{1}\right)-r\left(F_{1} F_{2} / F_{4}\right)^{1 / 2} \sin \phi_{+}-\delta_{+}^{\prime},
\end{gathered}
$$

where $H$ is the Hamiltonian of the system

$$
H=2\left(F_{1} F_{2}\right)^{1 / 2}\left(F_{3}^{1 / 2} \sin \phi_{-}-r F_{4}^{1 / 2} \sin \phi_{+}\right)-\delta_{-}^{\prime} F_{3}-\delta_{+}^{\prime} F_{4},
$$

and $\phi_{-}=\phi_{1}-\phi_{2}-\phi_{3}, \phi_{+}=\phi_{1}+\phi_{2}-\phi_{4} ; \delta_{\mp}^{\prime}=\delta_{\mp} / \omega_{p} ; \beta_{1}=\left(1 / c_{-}\right) \times$ $\left(2 m_{i} \omega_{2} / m_{e} \omega_{3}\right)^{1 / 2}, \beta_{2}=\left(\omega_{p} / c_{-}\right)\left(\omega_{1} \omega_{3}\right)^{-1 / 2}, \beta_{3}=\left(1 / c_{-}\right)\left(2 m_{i} \omega_{2} / m_{e} \omega_{1}\right)^{1 / 2} \times$ $\exp i \delta_{-}^{\prime} \tau$ and $\beta_{4}=\left(1 / c_{-}\right)\left(2 m_{i} \omega_{2} / m_{e} \omega_{1}\right)^{1 / 2} \exp i \delta_{+}^{\prime} \tau$; the dot denotes differentiation with respect to $\tau=\omega_{p} t$; the subscript 1 denotes the pump Langmuir wave, 2 the ion-acoustic daughter wave, 3 the Stokes Langmuir wave and 4 the anti-Stokes Langmuir wave.

In addition to the Hamiltonian given by Eq. (14), the set of equations (8)-(13) admits other conservation laws known as the Manley-Rowe relations:

$$
\begin{aligned}
& F_{1}+F_{3}+F_{4}=c_{1}, \\
& F_{2}-F_{3}+F_{4}=c_{2} .
\end{aligned}
$$

When the anti-Stokes mode $F_{4}$ is off-resonant, $c_{+} \rightarrow 0$ and $r \rightarrow 0$, the system given by Eqs. (8)-(13) degenerates to a three-wave decay process $L_{0} \rightleftharpoons$ $L_{-}+S$. Alternatively, when the Stokes mode $F_{3}$ is off-resonant, $c_{-} \rightarrow 0$ and $r \rightarrow \infty$, the system degenerates to a three-wave fusion process $L_{0}+S \rightleftharpoons L_{+}$.

If we specify $F_{3}(0)=F_{4}(0)=0$ as initial conditions, we have $H=0$ and $F_{4}(\tau)=r^{2} F_{3}(\tau)$. In the absence of frequency mismatch, $\delta_{-}^{\prime}=\delta_{+}^{\prime}=0$, we can obtain periodic analytical solutions of Eqs. (8)-(13). The solution for $F_{2}(\tau)$ is

$$
F_{2}(\tau)=c_{2}+\left(1-r^{2}\right) a_{1} \operatorname{cn}^{2}\left\{\left(a_{1}-a_{2}\right)^{1 / 2} \tau,\left[a_{1} /\left(a_{1}-a_{2}\right)\right]^{1 / 2}\right\}
$$

where $a_{1}=c_{1} /\left(1+r^{2}\right)$ and $a_{2}=c_{2} /\left(r^{2}-1\right)$; the solutions for $F_{1,3,4}$ can be determined from Eqs. (15)-(17). In the limit $r=1$, we have $F_{3}(\tau)=F_{4}(\tau)$, and it follows from Eq. (16) that $F_{2}=c_{2}=$ constant. The modulus of the cnoidal 
function in Eq. (17), $\left[a_{1} /\left(a_{1}-a_{2}\right)\right]^{1 / 2}$, becomes zero if $r=1$ since $a_{2}=\infty$ and the elliptic function becomes a sinusoidal function.

Let us now turn our attention to the transition from order to chaos of the Hamiltonian system described by Eqs. (8)-(13). In the special case $\delta_{-}^{\prime}=\delta_{+}^{\prime}=0$, the system of Eqs. (8)-(13) is integrable and the solutions are regular. We introduce a parameter $\Delta=\delta_{-}^{\prime}-\delta_{+}^{\prime}$ which is a measure of the relative linear frequency mismatch of the two wave triplets. When $\Delta \neq 0$ the system becomes nonintegrable and chaotic solutions may appear. The numerical solutions show that the finite value of $\Delta$ introduces new frequencies to the system. For a fixed initial condition, as $\Delta$ is increased, the periodic solutions become quasiperiodic and evolve to chaos. Windows of regular solutions within the chaotic regions due to phase locking have also been identified. The details of numerical solutions and characterization of ordered and chaotic solutions can be found in Chian et al. (1996).

In addition to the Langmuir stimulated modulational instability treated in this paper, transition from order to chaos in Langmuir turbulence also occurs in the Langmuir parametric decay instability (Lopes \& Chian 1996) and Langmuir oscillating two-stream instability (de Oliveira et al. 1995). Hence, deterministic chaos seems to be a common phenomenon in Langmuir turbulence.

In conclusion, we have shown that the nonlinear saturation of Langmuir stimulated modulational instability is governed by a set of coupled wave equations with quadratic nonlinearity. The saturated state of the Langmuir SMI can be either regular or chaotic. As the frequency mismatch parameter is varied, this Hamiltonian system can evolve from regular to chaotic states via the route of quasiperiodicity. Our study suggests that the periodic, quasiperiodic and chaotic behaviors in the AGN variability may be the manifestation of the corresponding orderly and chaotic dynamics of Langmuir turbulence in accretion disks or jets of AGN. It is likely that the analysis of the time series of AGN emissions will reveal these nonlinear dynamical features of AGN source region.

Acknowledgments. This research was supported by CNPq and FAPESP.

\section{References}

D. N. Baker, J. E. Borovsky, G. Benford, and J. A. Eilek 1988, ApJ 326, 110.

S. Bardwell and M. V. Goldman 1976, ApJ 209, 912.

A. C.-L. Chian 1996, ApSS, in press.

A. C.-L. Chian and M. V. Alves 1988, ApJ 330, L77.

A. C.-L. Chian and F. B. Rizzato 1994, J. Plasma Phys. 51, 61.

A. C.-L. Chian and J. R. Abalde 1995, A\&A, 298, L9.

A. C.-L. Chian, S. R. Lopes, and J. R. Abalde 1996, Physica D, in press.

G. I. de Oliveira, F. B. Rizzato, and A. C.-L. Chian 1995, Phys. Rev. E 52, 2025.

H. Lesch 1991, A\&A 245, 48.

S. R. Lopes and A. C.-L. Chian 1996, Phys. Rev. E 54, 170.

J. C. Weatherall, M. V. Goldman, and D. R. Nicholson 1981, ApJ 246, 306.

V. E. Zakharov 1972, Sov. Phys. JETP 35, 908. 